\title{
Microfluidic synthesis and post processing of non-spherical polymeric microparticles
}

\author{
David Baah $\cdot$ Julaunica Tigner • \\ Kala Bean · Nicole Walker • Bernard Britton • \\ Tamara Floyd-Smith
}

Received: 19 June 2011/ Accepted: 15 August 2011/Published online: 8 September 2011

(C) The Author(s) 2011. This article is published with open access at Springerlink.com

\begin{abstract}
Monodisperse spherical and non-spherical particles as well as their suspensions have diverse applications in optoelectronics, photonics, abrasives, catalysis, drug delivery, and field responsive rheological fluids. The synthesis of highly monodisperse particles with tunable functionalities has been a great challenge. Microfluidics technology, however, presents an attractive approach to synthesizing monodisperse non-spherical particles with tunable functionalities for application breakthroughs. The microfluidics method, described in a previous study, uses a UV-curable prepolymer with an appropriate photo-
\end{abstract}

Kala Bean, Nicole Walker and Bernard Britton are undergraduate researchers.

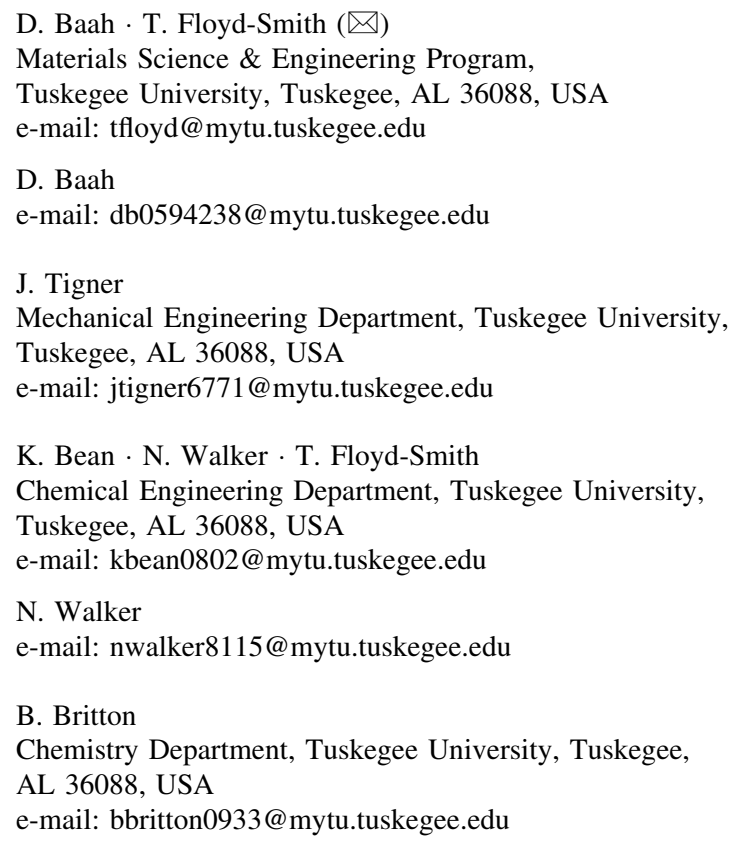

D. Baah · T. Floyd-Smith ( ( )

Materials Science \& Engineering Program,

Tuskegee University, Tuskegee, AL 36088, USA

e-mail: tfloyd@mytu.tuskegee.edu

D. Baah

e-mail: db0594238@mytu.tuskegee.edu

J. Tigner

Mechanical Engineering Department, Tuskegee University,

Tuskegee, AL 36088, USA

e-mail: jtigner6771@mytu.tuskegee.edu

K. Bean $\cdot$ N. Walker $\cdot$ T. Floyd-Smith

Chemical Engineering Department, Tuskegee University,

Tuskegee, AL 36088, USA

e-mail: kbean0802@mytu.tuskegee.edu

N. Walker

e-mail: nwalker8115@mytu.tuskegee.edu

B. Britton

Chemistry Department, Tuskegee University, Tuskegee,

AL 36088, USA

e-mail: bbritton0933@mytu.tuskegee.edu

initiator. The prepolymer solution passes through a microfluidic channel positioned on a microscope stage, and a microscope objective focuses UV light that is launched into the microfluidic channel. A photo mask patterned with transparent geometric features that define the shape of the particles masks the UV light to synthesize micron sized organic particles. Typically, particles synthesized using this method remain suspended. However, this study describes post-processing methods that allow the recovery of high fidelity, solvent-free particles. Particles in cubic, tetragonal and cylindrical shapes as well as those in pentagonal, hexagonal and triangular cross sections with a size range of $\sim 40-130 \mu \mathrm{m}$ were synthesized and collected. Then, they were characterized using electron microscopy and image processing to demonstrate the efficacy of the post processing techniques described.

Keywords Microfluidics - Particles - Photopolymer . Non-spherical

\section{Introduction}

In recent years, the synthesis of non-spherical nano and micro organic particles as well as their composites has attracted significant interest due to their myriad applications in the areas of optical light scattering, drug delivery, sensors, coatings and catalysis (Leite et al. 2000; Champion et al. 2007; Burda et al. 2005; Decuzzi et al. 2010; Yu et al. 2002). Equally exciting is the possible transformation of composite particles to ceramic particles through polymer burn off and consolidation (Conrad et al. 2011).

One application of monodisperse, non-spherical particles of particular interest to the authors is in the area of shape and size induced differential flow characteristics 
(viscosity) of suspensions, a key requirement of liquid body armor. The relationship between particle properties in a suspension and increasing viscosity has been a subject of numerous investigations (Barnes 1989; Bender and Wagner 1996; Vickers et al. 2009). According to Barnes (1989), particle shape is an important feature of shear thickening, the phenomenon that underpins liquid body armor. Barnes intimated that the effect of shear thickening with respect to particle shape follows the descending order of rods $>$ plates $>$ cubes/grains $>$ spheres when the same phase volume of particles is added to the liquid. Further investigation of the role of particle shape in shear thickening behavior would be aided by the development of dedicated particle synthesis techniques such as the one described in this study.

Whereas the use of solution reactions to generate complex shapes of nano and micro structures almost seems serendipitous, microfluidics technology offers a unique route toward synthesizing such complex, monodisperse particles with predetermined size and shape with relative ease (Takasi and Takeshi 2010; Helgeson et al. 2011; He et al. 2011). Thus, microfluidics technology is receiving increasing attention for synthesizing customized particles with spherical or non-spherical morphology. It is likely that the first demonstration of the use microfluidics to synthesize non-spherical micro organic structures using UV curable hydrogels was by Koh and Phisko (2003). Burdick et al. (2004) adopted their approach but with slight modification to fabricate films of hydrogel with gradients in cross-linking density and tethered molecules. Subsequently, the development of the protocol for synthesizing non-spherical particles and composite particles from photopolymerizable polymers using microfluidics technology has transitioned through continuous flow lithography (Dendukuri et al. 2006) and stop flow lithography (SFL) (Dendukuri et al. 2007) to the current state of a fully automated SFL compressed-air flow control system (Bong et al. 2011). The power of microfluidics in particle synthesis from photopolymerizable polymers is based on the ability to predetermine the size and shape of particles for specific application contrary to other well described methods such as microemulsion polymerization (LunaXavier et al. 2002; McDonald et al. 2000). Furthermore, polymeric particles as small as $1 \mu \mathrm{m}$ edge length have been synthesized using this method. Even smaller sizes in the range of hundreds of nanometers may be accessed by loading the polymeric particle with ceramic nanostructures, burning off the polymer and consolidating the ceramic material.

The core SFL technique, used in this study, is well established and holds great promise (Dendukuri et al. 2006, 2007; Hwang et al. 2009). However, one issue that arises is particle recovery (Helgeson et al. 2011). Particles are typically washed and remain in solution after synthesis. The primary novelty of this work is the demonstration of postprocessing for bulk collection of solvent-free, high fidelity particles prepared using the SFL method. In this paper, the authors demonstrate the efficacy of their post processing techniques by synthesizing high fidelity particles with selectable shape and tunable aspect ratios recovered in bulk and solvent-free. This demonstration represents a significant step forward in the area of SFL microfluidic synthesis of nonspherical particles pioneered by the Doyle Group at MIT.

\section{Experimental methods}

\subsection{Photomask design}

Adobe Illustrator CS4, computer software, is used to design the photomasks, and a high resolution printer prints the pattern in the form of a transparency film. A photomask of a single microfluidic channel is produced to fabricate the microfluidic device. For the particle synthesis, squares, pentagons, circles, triangles and hexagons are patterned on a transparency mask to produce particles using methods described below.

\subsection{Microfluidic device fabrication}

Microfluidic devices with suitable channels are fabricated using standard soft lithography methods previously described (McDonald and Whitesides 2002; Floyd-Smith et al. 2006; Baah et al. 2008). Briefly, the viscosity and spin coating speed of the SU-8 photo-resist control the depth of the microfluidic channel. After fabricating the SU-8 mold master on a silicon substrate in a clean room environment, poly(dimethylsiloxane) (PDMS Sylgard 184, Dow Corning) is cast on the mold master and cured at $\sim 70^{\circ} \mathrm{C}$ for at least $2 \mathrm{~h}$. The PDMS mold, containing the channel imprint, is detached from the SU-8 master and cut into $1.5 \mathrm{~cm}$ by $3.5 \mathrm{~cm}$ rectangles. Microscope slides are coated on one side with a thin film of PDMS which is half cured to facilitate bonding with the mold. Placing the mold and the prepared slide in conformal contact completes the microfluidic device and creates a hydrophobic channel to allow particles to suspend and flow in a hydrophilic medium after their formation.

\subsection{Particle synthesis}

The synthesis begins with 30-40 wt\% poly(ethylene glycol) diacrylate (PEG-DA, $M_{n}=575$ or 700, Sigma Aldrich) containing $1 \mathrm{wt} \%$ 2-hydroxy-2-methyl propiophenone as the photo initiator in water. The schematic in Fig. 1 illustrates the synthesis station. Patterned 
Fig. 1 Schematic of the manual stop flow lithography station including a microscope with a patterned mask inserted in the field stop area, a microfluidic device positioned on the microscope stage, a UV light source and a syringe pump to deliver fluids to the microfluidic channel. Inset Schematic of the automated sample supply system consisting of a threeway solenoid valve connected to a compressed-air source

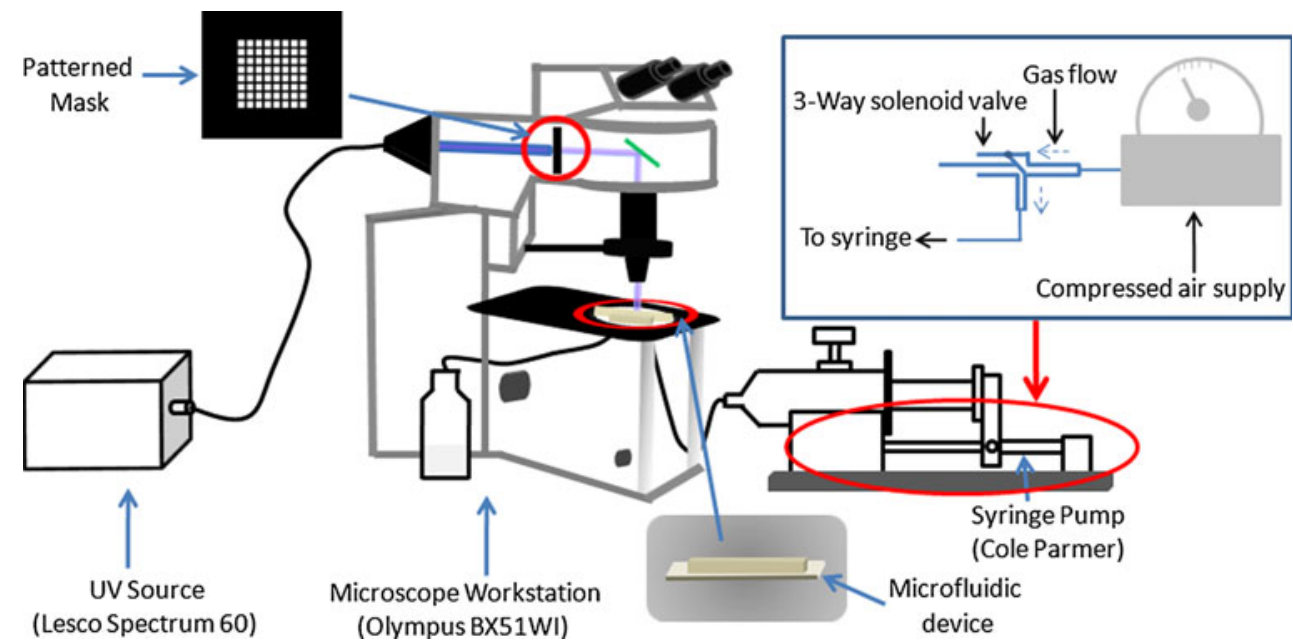

transparencies, inserted in the field stop area of an upright microscope (Olympus BX51WF Microscope), mask the UV light produced from a $60 \mathrm{~W}$ HBO mercury lamp source. Either a $10 \times$ or $20 \times$ objective focuses the UV light. For early experiments, a syringe pump (WPI SP220I, Cole Parmer) supplied reagents to the microfluidic device, and the solution stream was manually stopped prior to UV light exposure and re-started to flush out the particles. The exposure times were carefully chosen between 0.4 and $1.5 \mathrm{~s}$ depending on the pore size of the photomask, channel depth of the microfluidic device, and magnification of the objective lens used. The cycle was repeated until particle production goals were met, and the particles were separated from the unreacted prepolymer solution.

Recently, the implementation of a compressed-air flow control system (Bong et al. 2011) improved the synthesis efficiency tremendously. With this approach, the syringe pump is replaced with a compressed-air pumping method. In addition, the light source is replaced with a more intense Lumen 200 (Prior Scientific) equipped with a $200 \mathrm{~W}$ metal hydride bulb connected to a shutter driver (VCM-D1, Uniblitz). The opening and closure of the shutter driver is synchronized with that of the solenoid valve to control the introduction of fresh prepolymer solution as well as expelling synthesized particles. With this method, particles are synthesized as described above in 75-350 ms exposure times in a fully automated process.

Three different microfluidic devices with channel depths in the range of $60-80,100-150$ and $220-250 \mu \mathrm{m}$ are used to synthesize these particles with the aim of creating particles with different axial dimensions (aspect ratios). Square, hexagonal, pentagonal, triangular and circular cross-sectional shapes of particles are synthesized from the corresponding shape patterned in the photomask. The microscope objective magnification controls the particle cross sectional area.

\subsection{Particle post-processing}

The source of pre-polymer solution or sample supply using the fully automated system is a 5-ml syringe with the tip connected to the microfluidic device and a modified base connected to the solenoid valve. Controlled air pressure through the solenoid valve moves the sample from the syringe into the microfluidic device for UV exposure. This is a larger supply volume than the 5-125 $\mu$ l sample supply volume reported earlier by Bong et al. (2011). The collection tube is filled to half volume with distilled water which dilutes arriving particle-laden droplets of sample. The particle separation is purely by gravity settling because the particles stick to the wall of the tube when centrifuged and cannot be easily removed without being crushed. Stokes law indicates that the settling velocity $\left(v_{\mathrm{s}}\right)$ for a spherical particle is expressed by $v_{\mathrm{s}}=\frac{2}{9}\left(\frac{\rho_{\mathrm{p}}-\rho_{\mathrm{f}}}{\mu}\right) g R^{2}$ where $\rho_{\mathrm{p}}$ is the particle density, $\rho_{\mathrm{f}}$ the fluid density, $\mu$ the fluid viscosity, $g$ acceleration due to gravity and $R$ the radius of the particle. Based on Stokes law and assuming that the relationship holds for non-spherical particles, as expected, larger particles settle faster. Because the process is continuous, particles begin the settling process when they are expelled from the microfluidic channel into the collection tube. Typically, smaller particles $(<100 \mu \mathrm{m})$ settle within $20 \mathrm{~min}$ of synthesis completion and large particles $(>120 \mu \mathrm{m})$ settle within $5 \mathrm{~min}$ of synthesis completion.

After settling, the particles are subsequently collected by removing the supernatant and washing the particles of residual pre-polymer solution in deionized water. Drying is achieved by applying moderate vacuum to the particles in a dry seal desiccator. The moderate vacuum ensures structural integrity of the particles during drying. The dried particles, which can be re-suspended in an appropriate solvent for further processing, typically remain dry for 
imaging using either a Zeiss EVO 50VP or a JOEL JSM5800 scanning electron microscope.

\section{Results and discussion}

Using a $500 \mu \mathrm{m}$ pore size photomask and a $10 \times$ objective lens, cubic particles of edge dimension $130 \mu \mathrm{m}$ are synthesized (Fig. 2a). By changing the objective lens to $20 \times$, tetragonal-shaped particles with a comparable axial dimension but reduced cross-section of $50 \times 50 \mu \mathrm{m}$ are synthesized (Fig. 2b) demonstrating that the axial dimension or aspect ratio for particles of the same shape can be changed by changing the microscope objective. Figures 3a-c show hexagonal, circular, and triangular cross-section particles, respectively, that are synthesized and collected using the methods described. The particles are collected in bulk and solvent-free and imaged using scanning electron microscopy (SEM), a method tailored for high resolution morphology studies. Thus, collectively, Figs. 2 and 3 not only illustrate the power of microfluidics technology for synthesizing particles of tunable sizes and shapes, but also provide compelling evidence of the effectiveness of the post-processing techniques described.

In addition to qualitative characterization provided by SEM imaging, quantitative characterization was accomplished using image processing methods. Figure 4 shows the distribution of the axial dimension of the particles relative to the channel depth of the microfluidic devices used.
For the shallower channels $(60-80 \mu \mathrm{m})$, the particle extruded depth or average axial dimension ranged from 38 to $55 \mu \mathrm{m}$. For the $100-150 \mu \mathrm{m}$ channel depth, the average axial dimension ranged from 84 to $114 \mu \mathrm{m}$. Lastly, for the 200-250 $\mu \mathrm{m}$ channel depth, the particle axial dimension ranged from 131 to $139 \mu \mathrm{m}$. Even though, as expected, the particle's axial dimension increased with increasing channel depth, the sizes did not conform precisely to the $2.5 \mu \mathrm{m}$ oxygen inhibition layer reported by Dendukuri et al. (2006). Furthermore, it is unclear why the largest channels did not yield larger particles. Considering Beer's law, the light intensity as a function of depth $\left(I_{z}\right)$ is expressed by $I_{\mathrm{o}} \times 10^{-\alpha z}$ where $I_{\mathrm{o}}$ is the incident radiation, $\alpha$ the attenuation coefficient and $z$ the penetration depth. UV-Vis spectroscopy measurements were performed in a standard cuvette to determine the pre-polymer and polymer attenuation coefficients as well as to estimate the light penetration depth. The pre-polymer solution contains the monomer, the initiator and water. To determine the polymer parameters, the pre-polymer solution was polymerized in the cuvette. Removal of solid material from the cuvette after the polymerization confirmed that the polymerization was successful. For both sets of experiments, the absorbance values were $\sim 1.0$ for a $1 \mathrm{~cm}$ pathlength at $365 \mathrm{~nm}$, the initiator activation wavelength, suggesting that $\alpha \sim 1 \mathrm{~cm}^{-1}$. Assuming that $I_{z}$ is $95 \%$ of $I_{\mathrm{o}}$, Beer's law suggests a penetration depth of at least $200 \mu \mathrm{m}$ at $365 \mathrm{~nm} \pm 5 \mathrm{~nm}$, the only wavelengths transmitted through the filter. Thus, one would expect to achieve larger particles in the larger

Fig. 2 SEM micrographs of particles synthesized from a transparency film patterned with $500 \mu \mathrm{m}$ squares: a $10 \times$ objective yields cubes, b $20 \times$ objective yields high aspect ratio particles
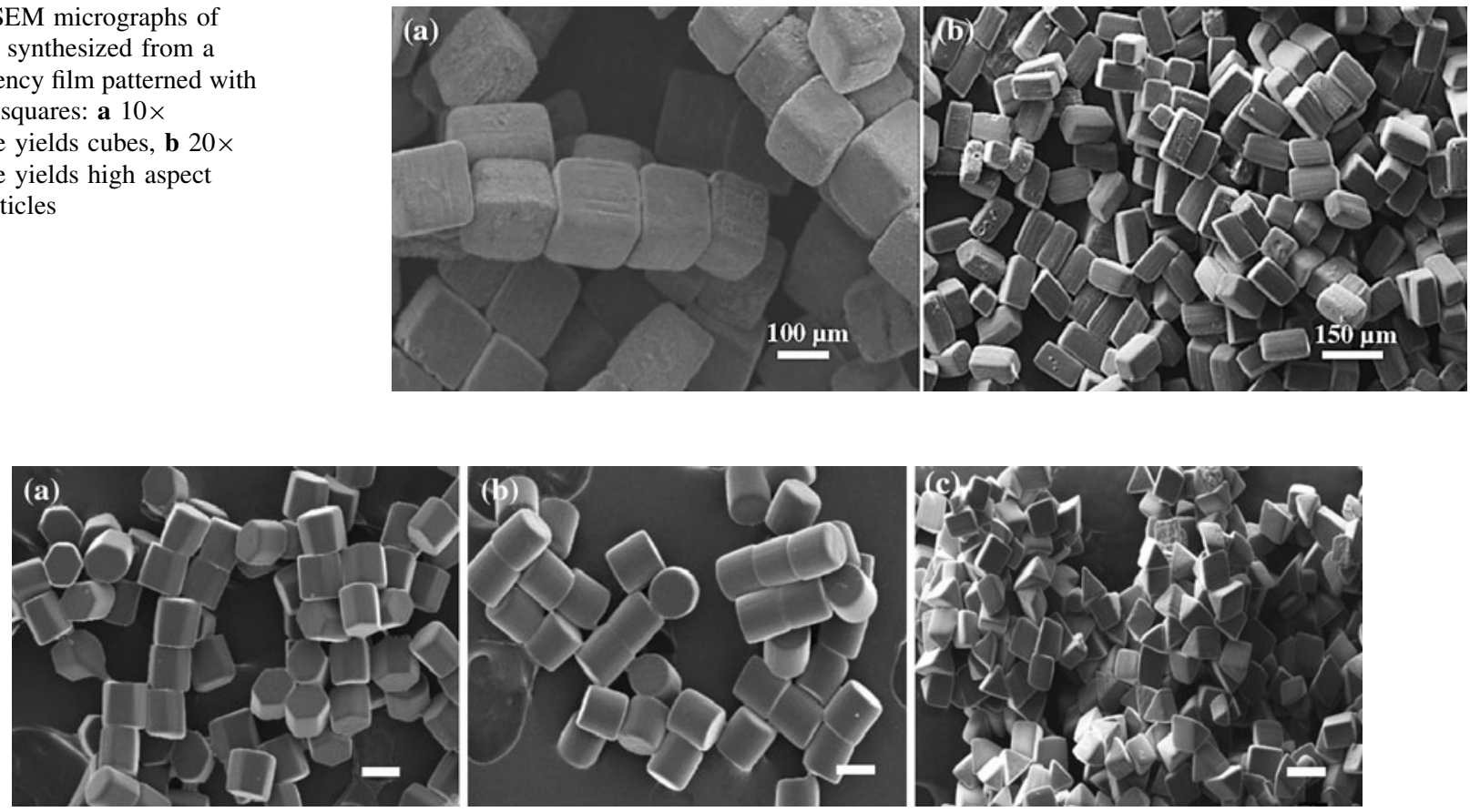

Fig. 3 SEM micrographs of non-spherical microparticles of varying cross section where the scale bar represents $100 \mu \mathrm{m}$ : a hexagonal, b circular and $\mathbf{c}$ triangular 
Fig. 4 Bar graph depicting the variation of particle axial dimension with microfluidic channel depth

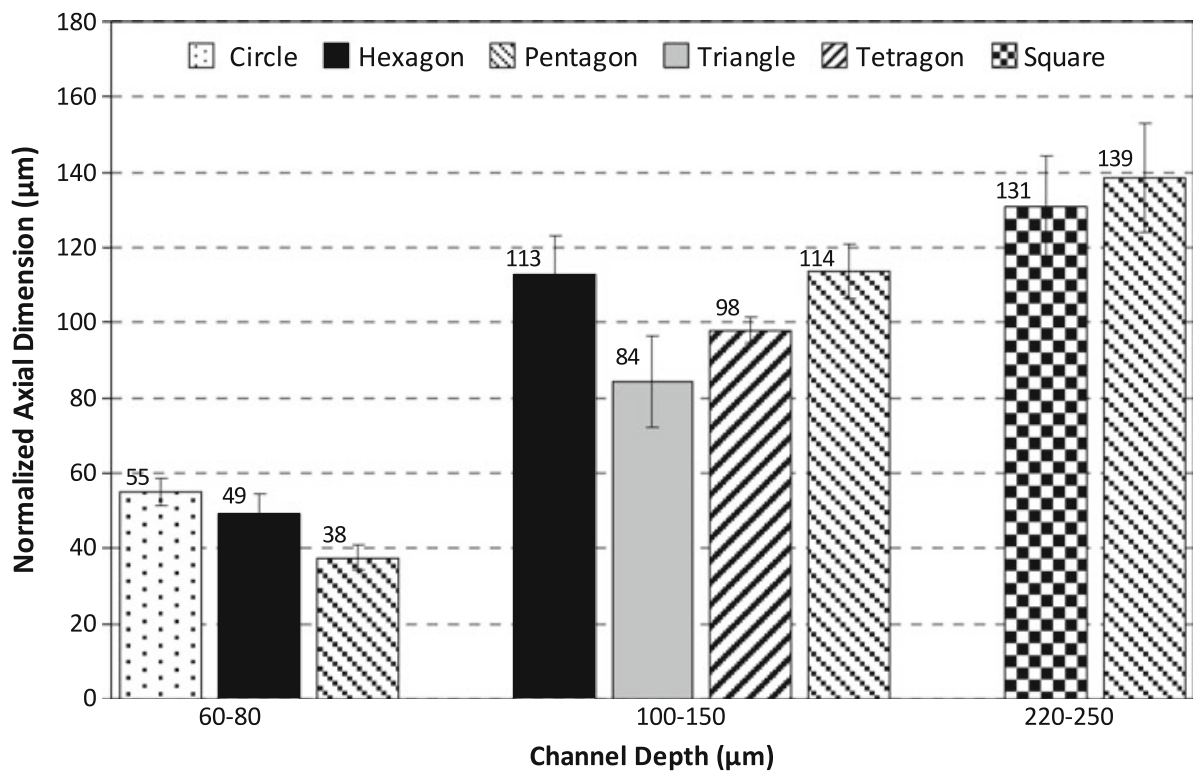

channels because the light intensity at the top of the channel, where polymerization always occurs, does not seem to be severely attenuated at the bottom of the channel by either the pre-polymer solution or polymer material that may have formed. More detailed studies in the future should clearly reveal the phenomena that determine the upper limits on the particle axial dimensions. Despite the unexpected plateau in the particle axial dimension, the small variations in the particle axial dimensions provide further evidence of the quality (one-dimensional monodispersity) of the particles produced using the post processing techniques described.

Figure 5 shows the average cross sectional area of three particles shapes synthesized using a transparency film with $500 \mu \mathrm{m}$ characteristic dimension pores. Clearly,

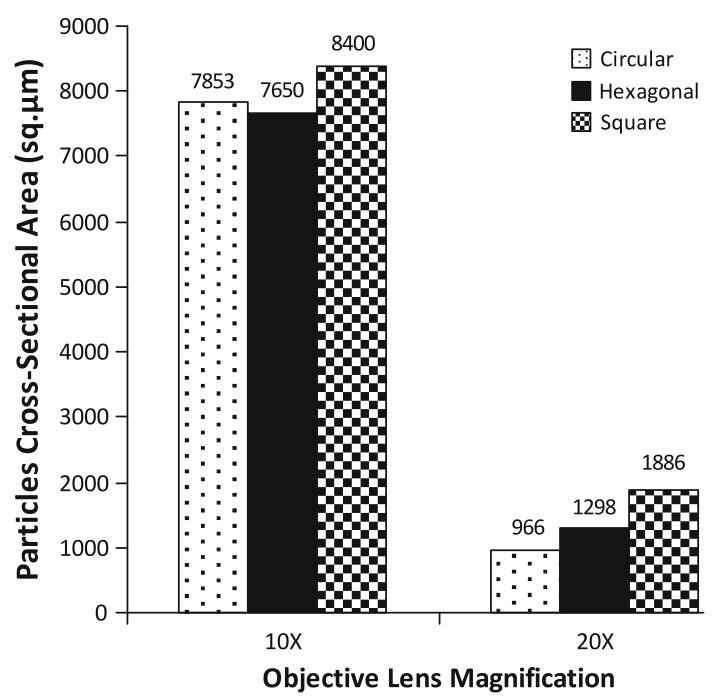

Fig. 5 Bar graph depicting the variation of particle cross sectional area with objective magnification microscope objective magnification controls the particle cross section where a change from $10 \times$ to $20 \times$ magnification yields particles that are an order of magnitude smaller in cross-sectional area. The error bars, not shown because they are so small that they are not clearly visible, provide additional evidence of the efficacy of the post processing techniques described which yield large quantities of solvent-free, high fidelity polymeric particles.

\section{Conclusions}

In this work, microfluidic methods for particle synthesis and post processing were described which produce high fidelity, solvent-free particles collected in bulk and imaged as such. Post processing methods include gravity separation to prevent particle damage caused by centrifugation and drying in a moderate vacuum after particle washing. The polymeric particles were characterized using SEM imaging and image analysis. SEM micrographs revealed good particle monodispersity and integrity. Image analysis provided additional quantitative evidence of the quality of the particles produced using the post processing methods described. Future work will include additional studies to understand the upper limits on particle size, synthesis of composite and ceramic particles using the techniques described and investigation of the rheological characteristics of suspensions of the particles synthesized using the core SFL method with the post processing techniques described.

Acknowledgments This research was supported by, or supported in part by, the US Army through the Institute for Soldier Nanotechnologies, under Contract DAAD-19-02-D-0002 with the US Army 
Research Office, and the National Science Foundation through the Grant DMR-0611612. Prof Patrick Doyle and his Research Group are acknowledged for helpful discussion and leadership with the system automation.

Open Access This article is distributed under the terms of the Creative Commons Attribution Noncommercial License which permits any noncommercial use, distribution, and reproduction in any medium, provided the original author(s) and source are credited.

\section{References}

Baah D, Vickers D, Hollinger A, Floyd-Smith T (2008) Patterned dispersion of nanoparticles in hydrogels using microfluidics. Mater Lett 62:3833-3835

Barnes HA (1989) Shear-thickening ("Dilatancy") in suspensions of nonaggregating solid particles dispersed in Newtonian liquids. J Rheol 33(2):329-366

Bender JW, Wagner NJ (1996) Reversible shear thickening in monodisperse and bidisperse colloidal dispersions. J Rheol 40(5):899-916

Bong KW, Chapin SC, Pregibon DC, Baah D, Floyd-Smith TM, Doyle PS (2011) Compressed-air flow control system. Lab Chip 11:743-747

Burda C, Chen X, Narayanan R, El-Sayed M (2005) Chemistry and properties of nanocrystals of different shapes. Chem Rev 105:1025-1102

Burdick JA, Khademhosseini A, Langer R (2004) Fabrication of gradient hydrogels using a microfluidics/photopolymerization process. Langmuir 20(13):5153-5156

Champion JA, Katare YK, Mitragotri S (2007) Particle shape: A new design parameter for micro- and nanoscale drug delivery carriers. J Control Release 121:3-9

Conrad JC, Ferreira SR, Yoshikawa J, Sheherd RF, Ahn BY, Lewis JA (2011) Designing colloidal suspensions for direct material assembly. Curr Opinion Coll Interface Sci 16:71-79

Decuzzi P, Godin B, Tanaka T, Lee SY, Chiappini C, Liu X, Ferreri $M$ (2010) Size and shape effects in the biodistribution of intravascularly injected particles. J Control Release 141:320-327
Dendukuri D, Pregibon DC, Collins J, Hatton TA, Doyle PS (2006) Continuous-flow lithography for high-throughput microparticle synthesis. Nat Mater 5:365-369

Dendukuri D, Gu S, Pregibon D, Hatton A, Doyle P (2007) Stop Flow lithography in a microfluidic device. Lab Chip 7:818-828

Floyd-Smith TM, Golden JP, Howell PB, Ligler FS (2006) Characterization of passive microfluidic mixers fabricated using soft lithography. Microfluid Nanofluid 2:180-183

He T, Liang Q, Zhang K, Mu X, Luo T, Wang Y, Luo G (2011) A modified microfluidic chip for fabrication of paclitaxel-loaded poly(L-lactic acid) microspheres. Microfluid Nanofluid 10: $1289-1298$

Helgeson MW, Chapin SC, Doyle PS (2011) Hydrogel microparticles from lithographic processes: Novel materials for fundamental and applied colloid science. Curr Opin Coll Interface Sci 16:106-117

Hwang DK, Oakey J, Toner M, Arthur JA, Anseth KS, Lee S, Zeiger A, van Vliet KJ, Doyle PS (2009) Stop-flow lithography for the production of shape evolving degradable microgel particles. J Am Chem Soc 131(12):4499-4504

Koh WG, Phisko M (2003) Photoreaction injection molding of biomaterial microstructures. Langmuir 19:10310-10316

Leite ER, Weber IT, Longo E, Velara JA (2000) A new method to control particle size and particle size distribution of $\mathrm{SnO}_{2}$ nanoparticles for gas sensor applications. Adv Mater 12:965-968

Luna-Xavier LJ, Guyot A, Bourgeat-Lami E (2002) Synthesis and characterization of silica/poly(methyl metharcrylate) nanocomposite latex particles through emulsion polymerization using a Cationic Azo Initiator. J Colloid Interface Sci 250:82-92

McDonald CJ, Whitesides GM (2002) Poly(dimethylsiloxane) as material for fabricating devices. Acc Chem Res 35:491-499

McDonald CJ, Bouck KJ, Chaput AB (2000) Emulsion polymerization of voided particles by encapsulation of a nonsolvent. Macromolecules 33:1593-1605

Takasi N, Takeshi H (2010) A microfluidic cross-flowing emulsion generator for producing biphasic droplets and anisotropically shaped polymer particles. Microfluid Nanofluid 9:427-437

Vickers D, Archer LA, Floyd-Smith T (2009) Synthesis and characterization of cubic cobalt oxide nanocomposite fluids. Colloids Surf A 348:39-44

$\mathrm{Yu}$ JC, Zhang L, Yu J (2002) Rapid synthesis of mesoporous $\mathrm{TiO}_{2}$ with high photocatalytic activity by ultrasound-induced agglomeration. New J Chem 26:416-420 